\title{
Complémentation d'un ensilage d'herbe par des pulpes de betteraves : effet sur les quantités ingérées, les activités alimentaires et méryciques, et la digestion chez les génisses
}

\author{
P Kamatali *, E Teller, M Vanbelle, \\ $P$ Delfosse, M Foulon, G Collignon \\ Université Catholique de Louvain, AGRO/BNUT, Place Croix du Sud 3, \\ 1348 Louvain-la-Neuve, Belgique
}

(Reçu le 19 février 1990, accepté le 7 juin 1990)

\begin{abstract}
Résumé - Un ensilage d'herbe préfanée de coupe tardive a été distribué seul ou supplémenté avec $2,56 \mathrm{~kg}$ de matière sèche de pulpes sèches de betteraves, à 6 génisses de race Frisonne selon un schéma expérimental avec inversion. Les 2 rations étaient complémentées par $1 \mathrm{~kg}$ de tourteau de soja.

La supplémentation avec les pulpes sèches a amélioré l'ingestion de matière sèche totale $(11,7$ contre $10,7 \mathrm{~kg}$ de MS ingérée par jour pour l'ensilage non complémenté). Le taux de substitution des pulpes sèches à l'ensilage a été de $0,64 \mathrm{~kg}$ de matière sèche $/ \mathrm{kg}$ de $\mathrm{MS}$ de pulpe apportée.

La durée journalière de rumination a été réduite par la supplémentation avec des pulpes sèches ( 552 contre $584 \mathrm{~min} / \mathrm{j})$, de même que la durée unitaire de rumination $(47,9$ contre $54,6 \mathrm{~min}$ par $\mathrm{kg}$ de MS ingérée) et la durée unitaire de mastication (81,2 contre 96,7 min par $\mathrm{kg}$ de matière sèche ingérée). La durée de mastication par $\mathrm{kg}$ de MS du supplément de pulpes a été de $\mathbf{4 5} \mathrm{min}$, conférant ainsi à cet aliment un indice de fibrosité très appréciable.

L'adjonction de pulpes sèches à de l'ensilage d'herbe a augmenté le pourcentage molaire d'acide butyrique $(12,1$ contre $10,5 \%)$ et réduit la concentration ammoniacale $(98,5$ contre $132,6 \mathrm{mg} /)$ dans le contenu ruminal. En même temps, la quantité d'azote total et celle d'acides aminés à l'entrée de l'intestin grêle ont été augmentées, ce qui s'explique par une synthèse plus importante de protéines bactériennes $(+27 \%)$.

La digestibilité de la matière organique $(59,6$ contre $51,2 \%$ ) et la valeur alimentaire de la ration ont été améliorées par suite de la complémentation de l'ensilage d'herbe par des pulpes. Ces dernières apparaissent donc comme un aliment à valeur énergétique élevée tout en ayant un indice de fibrosité important.
\end{abstract}

bovin / ensilage d'herbe / pulpe de betteraves / ingestibilité / comportement alimentaire / digestibilité

Summary - The addition of sugar beet pulp to late-cut wilted grass silage: effect on voluntary intake, chewing behavior and digestion by heifers. Six nonpregnant Friesian heifers with ruminal and duodenal cannulae were used to examine the effect of dried sugar beet pulp $(2.56 \mathrm{~kg} \mathrm{DM} /$ d) on voluntary intake, chewing behavior and digestion of late-cut wilted grass silage. Both diets were supplemented with $1 \mathrm{~kg}$ soybean meal. The animals were allotted according to a cross-over design.

\footnotetext{
* Correspondance et tirés à part.
} 
The pulp increased total DM intake (11.7 versus $10.7 \mathrm{~kg} D M / d$ of the non-supplemented silage). The rate of substitution of the pulp for silage DM was $64 \%$.

Daily duration spent ruminating was reduced (552 versus $584 \mathrm{~min} / \mathrm{d}$ ); the unitary duration of ruminating (47.9 versus $54.6 \mathrm{~min} / \mathrm{kg} \mathrm{DM}$ ) and total mastication (81.2 versus $96.7 \mathrm{~min} / \mathrm{kg} \mathrm{DM}$ ) also diminished with the pulp. The roughage value index (duration of chewing $/ \mathrm{kg} D M$ ) of the dried sugar beet pulp was $45 \mathrm{~min}$.

Supplementation of the grass silage with dried pulp slightly increased the percentage of butyric acid in ruminal content (12.1 versus $10.5 \%$ ) and reduced the ammonia concentration (98.5 versus 132.6 $\mathrm{mg} / 1)$. At the same time, the amounts of total nitrogen and amino acids in duodenal digesta were enhanced, which was mainly due to the improved bacterial protein synthesis in the rumen $(+27 \%)$.

The apparent digestibility of organic matter (59.6 versus $51.2 \%$ ) and, as a consequence, the energy supply of the diet increased when the silage was supplemented with dried pulp. It was concluded that, although dried sugar beet pulp has a high energy value, this feed also appears as an excellent stimulant of chewing activity.

bovine / grass silage / beet pulp / intake / eating behavior / digestibility

\section{INTRODUCTION}

Les pulpes sèches de betteraves sucrières sont un coproduit de haute valeur nutritive et peuvent constituer une partie importante de la ration tant des vaches laitières (Castle, 1972; Vanbelle, 1973; Dulphy et al, 1978; De Brabander et al, 1980) que des bovins à l'engrais (Buysse et Boucqué, 1967; Cordiez et al, 1977). L'ensilage de pulpes humides occasionne de grandes pertes de conservation alors que les coûts de déshydratation rendent la pulpe sèche moins compétitive en tant que produit de substitution partielle des fourrages grossiers (De Brabander et al, 1980).

Les pulpes sèches restent toutefois intéressantes en tant que composants des aliments concentrés pour les bovins. Elles ont une haute valeur énergétique grâce à leur teneur en constituants pariétaux $(49 \%)$ très digestibles (Welch et Smith, 1971). En effet, on peut dire que $1 \mathrm{~kg}$ de pulpes sèches équivaut à $1 \mathrm{~kg}$ d'orge pour la production laitière (Bhattacharya et Sleiman, 1971; Castle, 1972; Vanbelle, 1973) et pour l'engraissement (Cordiez et al, 1977); mais Buysse et Boucqué (1967) si- tuent la valeur des pulpes à $85-87 \%$ de celle de l'orge concassée contenant $5 \%$ de fibres brutes pour l'engraissement de bovins.

Malgré l'utilisation très répandue des pulpes sèches dans les rations pour la production laitière et de viande, des questions persistent quant à l'effet de ces aliments sur les processus fermentaires dans le rumen et, en particulier, sur la digestibilité partielle des nutriments à ce niveau. De plus, les répercussions de la substitution de pulpes sèches à de l'ensilage d'herbe sur les activités alimentaires et méryciques des animaux sont encore mal connues. En d'autres termes, quel indice de fibrosité et quelle valeur d'encombrement peut-on attribuer à cet aliment ? Le présent travail, avec des génisses nourries à l'ensilage d'herbe préfanée récoltée à un stade tardif, a pour but d'aborder ce problème et d'étudier l'influence des pulpes sèches de betteraves sur l'ingestion volontaire de matière sèche, sur les activités alimentaires et méryciques, sur les fermentations et la digestion partielle dans le réticulo-rumen, ainsi que sur la digestion dans le tractus digestif total. 


\section{MATÉRIEL ET MÉTHODES}

\section{Animaux}

Six génisses non gestantes de race Frisonne, âgées de 47 mois et pesant $524 \mathrm{~kg}$ au démarrage de l'essai, ont été pourvues d'une canule ruminale et d'une canule en $T$ dans le duodénum proximal. Elles ont été réparties selon un schéma expérimental avec inversion $(2 \times 2 \times 6)$, à savoir 2 traitements (ensilage d'herbe préfanée de coupe tardive apporté à volonté, supplémenté ou non par des pulpes sèches de betteraves), 2 périodes de 26 j et 6 génisses.

\section{Aliments distribués}

Les rations distribuées consistaient en un ensilage d'herbe préfanée de coupe tardive offert ad libitum complémenté par $1 \mathrm{~kg}$ de tourteau de soja (ration témoin), ou du même ensilage ad libitum complémenté par $1 \mathrm{~kg}$ de tourteau de soja plus $3 \mathrm{~kg}$ de pulpes sèches (ration expérimentale).

L'ensilage a été réalisé à partir d'une prairie permanente de premier cycle dont la composition florale était : Lolium perenne $(81 \%)$, Poa pratensis L (9\%), Phleum pratense (5\%) et Poa trivialis, Poa annua, Taraxacum officinalis et Equisetum arvense (5\%). L'herbe a été fauchée, séchée pendant $40 \mathrm{~h}$ au sol, hachée en brins courts $(1,8 \mathrm{~cm})$ au moyen d'une ensileuse à 9 couteaux (New Holland) et ensilée sans conservateur en silo taupinière. La composition chimique des aliments distribués et les caractéristiques fermentaires de l'ensilage sont données au tableau I.

L'ensilage était pauvre en azote total (16 g de $\mathrm{N}$ total/kg de MS d'ensilage) dont $61 \%$ solubles; le $\mathrm{pH}$ était de 4,39 et les teneurs en acides acétique, butyrique et lactique étaient de $16 \mathrm{~g}$, de $0 \mathrm{~g}$ et de $77 \mathrm{~g} / \mathrm{kg}$ de MS, respectivement.

Ce fourrage était distribué en 2 repas à $9 \mathrm{~h}$ et à $16 \mathrm{~h}$, et les animaux disposaient en permanence d'eau de boisson et de pierres à lécher, d'un mélange de minéraux et de vitamines. La moitié de la quantité journalière d'aliment concentré, c'est-à-dire $0,5 \mathrm{~kg}$ de soja pour la ration témoin et $0,5 \mathrm{~kg}$ de soja $+1,5 \mathrm{~kg}$ de pulpes sèches pour la ration complémentée, était offerte avant chaque distribution du fourrage grossier.

\section{Le comportement alimentaire et mérycique}

La méthode d'enregistrement et les unités de mesure utilisées ont été, respectivement, celles de Ruckebusch (1963) et de Dulphy (1971) :

Tableau I. Composition chimique des aliments (en $\mathrm{g} / \mathrm{kg}$ MS excepté autres indications).

\begin{tabular}{lccc}
\hline & $\begin{array}{c}\text { Ensilage d'herbe } \\
\text { préfanée }\end{array}$ & $\begin{array}{c}\text { Tourteau de soja } \\
\text { déshuilé }\end{array}$ & $\begin{array}{c}\text { Pulpes sèches } \\
\text { de betteraves }\end{array}$ \\
\hline & & & \\
Matière sèche $(\mathrm{g} / \mathrm{kg})$ & 481 & 860 & 856 \\
Matière organique & 912 & 77 & 916 \\
$\mathrm{~N}$ total & 16 & 120 & 17 \\
$\mathrm{Cellulose}$ brute & 318 & 185 & 224 \\
$\mathrm{NDF}$ & 585 & 16 & 10 \\
Matière grasse & 18 & & \\
$\mathrm{~N}$ soluble $(\% \mathrm{~N}$ total) & 61 & & \\
$\mathrm{~N}-\mathrm{NH} \mathrm{H}_{3}(\% \mathrm{~N}$ total) & 11 & & \\
pH & 4,39 & & \\
Acide acétique & 16 & & \\
Acide butyrique & 0 & & \\
Acide lactique & 77 & & \\
Dimension moyenne des & & & \\
brins d'ensilage $(\mathrm{cm})$ & 1,84 & & \\
\hline
\end{tabular}


- les durées journalières d'ingestion et de rumination correspondent à toutes les activités d'ingestion et de rumination durant $24 \mathrm{~h}$;

- les durées unitaires d'ingestion (DUI) et de rumination (DUR) correspondent au temps passé à ingérer ou à ruminer chaque $\mathrm{kg}$ de MS ingérée;

- le repas (ou période de rumination) correspond à une durée d'ingestion (ou de rumination) au moins égale ou supérieure à $7 \mathrm{~min}$. Le repas principal est celui qui suit l'affouragement. La pose des harnais a été effectuée $2 \mathrm{j}$ avant le début des enregistrements continus des mouvements de mâchoires pendant 5 j.

\section{Caractéristiques du contenu du rumen}

Huit prélèvements de $100 \mathrm{ml}$ de liquide du rumen ont été effectués, le premier avant le repas du matin et les autres à des intervalles de $1 \mathrm{~h}$.

Les échantillons ont été centrifugés à 1000 tours $/ \mathrm{min}$, pendant $10 \mathrm{~min}$ et le surnageant a été utilisé pour déterminer le $\mathrm{pH}$, l'azote ammoniacal $\left(\mathrm{N}-\mathrm{NH}_{3}\right.$ ) et les acides gras volatils (AGV) selon les méthodes indiquées par Teller et al (1989).

Dans ces mêmes échantillons de contenu ruminal, le polyéthylèneglycol-4000 (PEG) a été dosé selon la méthode de Hyden (1955). La vitesse de diminution de la concentration du PEG a été utilisée pour calculer le taux de renouvellement de la phase liquide selon la formule décrite par Faichney (1975) :

$$
C=C_{0} \cdot e^{-k t}
$$

avec $C=$ concentration au temps $t ; C_{0}$ : concentration initiale; $k$ : taux de renouvellement de la phase liquide dans le rumen.

\section{Flux duodénaux}

L'oxyde de chrome $\left(\mathrm{Cr}_{2} \mathrm{O}_{3}\right)$ et le polyéthylèneglycol (PEG) ont été utilisés pour mesurer les flux des digesta duodénaux. Ainsi, $20 \mathrm{~g}$ de $\mathrm{Cr}_{2} \mathrm{O}_{3}$ et $90 \mathrm{~g}$ de PEG par jour ont été introduits en 2 fois ( $8 \mathrm{~h} 30$ et $16 \mathrm{~h} 30$ ) par la canule rumi- nale. Le prélèvement des digesta duodénaux a été effectué à raison de $300 \mathrm{~g}$ toutes les $2 \mathrm{~h}$ pendant toute la période nycthémérale. Le flux de digesta correspond à la moyenne des flux obtenus sur base du PEG et du $\mathrm{Cr}_{2} \mathrm{O}_{3}$ respectivement, selon la méthode décrite par Madsen et Hvelplund (1985). Le chrome a été déterminé selon la méthode décrite par Petry et Rapp (1971). L'azote endogène a été estimé à $2,7 \mathrm{~g}$ par $\mathrm{kg}$ de matière sèche affluant au duodénum (Narasimhalu et al, 1989).

\section{Digestibilités apparentes}

Les digestibilités apparentes des nutriments dans le tractus digestif total ont été déterminées au moyen d'un marqueur externe : l'oxyde de chrome $\left(\mathrm{Cr}_{2} \mathrm{O}_{3}\right)$.

Les prélèvements de matières fécales furent effectués directement au niveau du rectum, 2 fois par jour $(8 \mathrm{~h} 30$ et $16 \mathrm{~h} 30)$ pendant $6 \mathrm{j}$ à raison de $500 \mathrm{~g}$ par prélèvement. Les quantités de matières fécales émises par j ont été estimées à partir de la concentration en $\mathrm{Cr}_{2} \mathrm{O}_{3}$ par rapport a la dose journalière administrée (Bartiaux-Thill et al, 1988).

\section{Analyses chimiques}

L'analyse des aliments, des digesta duodénaux et des matières fécales a été réalisée sur des échantillons lyophilisés. Pour les ensilages, la perte en éléments volatils a été prise en considération (Schoch, 1949).

La dimension moyenne des particules d'ensilage a été déterminée par classification manuelle des brins d'un échantillon d'ensilage en catégories de différentes longueurs.

Les matières grasses ont été déterminées par extraction à froid au tétrachlorure de carbone, la teneur en azote selon la méthode Kjeldahl standardisée (AOAC, 1960) et l'azote soluble après incubation de $100 \mathrm{~g}$ de matière fraîche dans 1 / d'eau distillée, pendant $24 \mathrm{~h}$. L'ammoniac a été dosé selon la méthode Whitehead et al (1967), les parois végétales (NDF) selon la méthode de Goering et Van Soest (1970). Cette teneur dans le soja et les pulpes a été déterminée après le traitement à l'amyłase selon la méthode de Giger et al (1987). 
La pression osmotique du jus du rumen a été déterminée sur un osmomètre de précision (Osmette, Precision Systems, Inc, Newton, MA), le $\mathrm{pH}$ au moyen d'un pHmètre à électrode de verre et l'acide diaminopimélique (DAPA) selon la méthode décrite par El-Shazly et Hungate (1966).

\section{Analyse des résultats}

Les résultats expérimentaux ont été traités statistiquement selon la méthode d'analyse ANOVA (Cochran et Cox, 1957) en utilisant la procédure GLM de SAS (1982) pour le schéma en inversion. Les 11 degrés de liberté (dl) étaient répartis selon le traitement (1 dl), la période (1 dl), les génisses $(5 \mathrm{dl})$ et la variance résiduelle (4 di) (Teller et al, 1989).

\section{RÉSULTATS}

\section{Ingestion et rumination}

Les quantités ingérées journalières de MS totale (tableau II) ont été plus élevées $(P<$ $0,01)$ avec la ration expérimentale $(+8 \%)$.

Tableau II. Effet de la complémentation de l'ensilage d'herbe par des pulpes sèches sur les quantités ingérées, les gains de poids vif́s moyens et les activités alimentaires et méryciques chez les génisses.

\begin{tabular}{|c|c|c|c|c|}
\hline Rations & $\begin{array}{l}\text { Ration } \\
\text { témoin }\end{array}$ & $\begin{array}{c}\text { Ration } \\
\text { expérimentale }\end{array}$ & $E T$ & $\begin{array}{l}\text { Niveau } \\
\text { signif stat }\end{array}$ \\
\hline $\begin{array}{l}\text { Quantités ingérées (kg MS/j) } \\
\text { - Ensilage } \\
\text { - Pulpes sèches } \\
\text { - Tourteau de soja } \\
\text { Total }\end{array}$ & $\begin{array}{r}9,9 \\
0,8 \\
10,7\end{array}$ & $\begin{array}{r}8,3 \\
2,6 \\
0,8 \\
11,7\end{array}$ & $\begin{array}{l}0,3 \\
0,3\end{array}$ & $x x$ \\
\hline Poids vif moyen $(\mathrm{kg})$ & 567 & 568 & 2,3 & NS \\
\hline Gain de poids moyen (kg/j) & 1,27 & 1,19 & 0,13 & NS \\
\hline $\begin{array}{l}\text { Durées totales d'ingestion } \\
\text { (min/j) } \\
\text { (min } / \mathrm{kg} \text { MS ingérée) }\end{array}$ & $\begin{array}{l}448 \\
42,2\end{array}$ & $\begin{array}{l}385 \\
33,3\end{array}$ & $\begin{array}{l}21 \\
2,6\end{array}$ & $\begin{array}{l}\text { NS } \\
\text { NS }\end{array}$ \\
\hline $\begin{array}{l}\text { Durées d'ingestion de l'ensilage seul } \\
(\mathrm{min} / \mathrm{j}) \\
(\mathrm{min} / \mathrm{kg} \text { MS ingérée) }\end{array}$ & $\begin{array}{l}423 \\
43,9\end{array}$ & $\begin{array}{l}347 \\
45,2\end{array}$ & 20 & $\begin{array}{l}\text { NS } \\
\text { NS }\end{array}$ \\
\hline $\begin{array}{l}\text { Durées totales de rumination } \\
\text { (min/j) } \\
(\mathrm{min} / \mathrm{kg} \text { MS ingérée) }\end{array}$ & $\begin{array}{l}584 \\
54,6\end{array}$ & $\begin{array}{l}552 \\
47,9\end{array}$ & $\begin{array}{l}7,1 \\
1,3\end{array}$ & $\begin{array}{l}x \\
x\end{array}$ \\
\hline Durée moyenne d'un cycle de rumination (sec) & 48 & 46 & 0,7 & NS \\
\hline $\begin{array}{l}\text { Nombre unitaire de cycles de rumination } \\
\text { (cycles/kg MS ingérée) }\end{array}$ & 68 & 62 & 1,3 & $x x$ \\
\hline $\begin{array}{l}\text { Durées de mastication } \\
(\mathrm{min} / \mathrm{j}) \\
(\mathrm{min} / \mathrm{kg} \text { MS ingérée) }\end{array}$ & $\begin{array}{l}1032 \\
96,7\end{array}$ & $\begin{array}{l}938 \\
81,2\end{array}$ & $\begin{array}{r}15,7 \\
3,4\end{array}$ & $\begin{array}{l}x \\
x\end{array}$ \\
\hline
\end{tabular}

$\mathrm{x}: P<0,05 ; \mathrm{xx}: P<0,01 ; \mathrm{xxx}: P<0,001 .{ }^{1}$ Entre les deux traitements. 
En revanche, l'ingestion volontaire de l'ensilage d'herbe pris séparément est réduite de $22 \%(P<0,001)$. L'apport de $2,56 \mathrm{~kg}$ de matière sèche de pulpes sèches par jour a entraîné une réduction de l'ingestion d'ensilage d'herbe de $1,6 \mathrm{~kg}$ de matière sèche, soit un taux de substitution de 0,64 $\mathrm{kg}$ de $\mathrm{MS} / \mathrm{kg}$ de MS de pulpe sèche apportée. Les gains de poids vif moyen sont comparables pour les 2 rations.

Les durées journalières d'ingestion (min/j) des 2 types de rations n'ont pas différé de manière significative (tableau II), bien qu'une tendance soit apparue en faveur d'une ingestion plus rapide de la ration expérimentale $(-9 \mathrm{~min} / \mathrm{kg}$ MS ingérée). La vitesse d'ingestion de l'ensilage pris séparément (min/ $\mathrm{kg}$ MS ingérée) n'a pas été affectée par la distribution des pulpes sèches.

La substitution des pulpes à l'ensilage a sensiblement réduit $(P<0,05)$ la durée journalière de rumination $(-32 \mathrm{~min} / \mathrm{j})$ et la durée unitaire de rumination de la matière sèche ingérée (-6,7 $\mathrm{min} / \mathrm{kg}$ MS ingérée).

La durée moyenne d'un cycle de rumination a été semblable pour les 2 types de rations, mais le nombre unitaire de cycles de rumination a diminué $(P<0,05)$ par suite de la complémentation de l'ensilage par des pulpes sèches. La durée journalière et la durée unitaire de mastication ont été réduites $(P<0,05)$ par la supplémentation avec des pulpes.

\section{Digestion}

Les caractéristiques du contenu ruminal n'ont pas été différentes, sauf pour l'acide butyrique dont le pourcentage molaire était plus faible $(P<0,01)$ avec la ration témoin (tableau III). La concentration en ammoniac dans le liquide du rumen a été plus faible $(P<0,05)$ pour la ration complémentée $(-26 \%)$. La pression osmotique et le taux de dilution de la phase liquide du rumen ont été semblables pour les 2 rations.

La complémentation avec les pulpes sèches n'a pas affecté de manière significative les digestibilités apparentes de la MS et de la matière organique dans le rumen (tableau IV), bien qu'une nette ten-

Tableau III. Effet de la complémentation de l'ensilage d'herbe par des pulpes sèches sur les caractéristiques du liquide du rumen chez des génisses.

\begin{tabular}{lcccc}
\hline & $\begin{array}{c}\text { Ration } \\
\text { témoin }\end{array}$ & $\begin{array}{c}\text { Ration } \\
\text { expérimentale }\end{array}$ & $E T$ & $\begin{array}{c}\text { Niveau } \\
\text { signif stat }{ }^{1}\end{array}$ \\
\hline $\mathrm{pH}$ & 6,54 & 6,37 & 0,12 & $\mathrm{NS}$ \\
Pression osmotique (mOsm/l) & 292 & 292 & 0,1 & $\mathrm{NS}$ \\
Acides gras volatils totaux (mmol/) & 91 & 96 & 3,1 & $\mathrm{NS}$ \\
Acide acétique (\% molaire) & 70 & 69 & 0,3 & $\mathrm{NS}$ \\
Acide propionique (\% molaire) & 20 & 19 & 0,4 & $\mathrm{NS}$ \\
Acide butyrique (\% molaire) & 11 & 12 & 0,2 & $\mathrm{xx}$ \\
$\mathrm{NH}_{3}$ (mg/) & 133 & 99 & 5,3 & $\mathrm{x}$ \\
$\begin{array}{l}\text { Taux de dilution de la phase liquide } \\
\text { du rumen (h-1) }\end{array}$ & 0,15 & 0,16 & 0,01 & $\mathrm{NS}$ \\
& & & &
\end{tabular}

$\mathrm{x}: P<0,05 ; \mathrm{xx}: P<0,01 .{ }^{1}$ Entre les 2 traitements. 
Tableau IV. Effet de la complémentation du fourrage par des pulpes sèches sur les digestibilités apparentes dans le rumen et dans le tractus digestif total.

\begin{tabular}{lcccc}
\hline & $\begin{array}{c}\text { Ration } \\
\text { témoin }\end{array}$ & $\begin{array}{c}\text { Ration } \\
\text { expérimentale }\end{array}$ & $E T$ & $\begin{array}{c}\text { Niveau } \\
\text { signif stat }{ }^{1}\end{array}$ \\
\hline $\begin{array}{l}\text { Digestibilités apparentes } \\
\text { partielles dans le rumen (\%) : } \\
\text { de la matière sèche } \\
\text { de la matière organique }\end{array}$ & 33,3 & 40,5 & 2,7 & $\mathrm{NS}$ \\
$\begin{array}{l}\text { Digestibilités apparentes } \\
\text { dans le tractus digestif total (\%) }\end{array}$ & 45,2 & 51,3 & 2,4 & $\mathrm{NS}$ \\
$\begin{array}{l}\text { de la matière sèche } \\
\text { de la matière organique }\end{array}$ & 47,4 & 54,8 & 1,0 & $\mathrm{Xx}$ \\
& 51,6 & 59,6 & 0,9 & $\mathrm{xx}$ \\
\hline
\end{tabular}

$x x: P<0,01,{ }^{1}$ Entre les 2 traitements.

dance soit apparue en faveur de la ration complémentée par les pulpes sèches ( $+7,2$ et $+6,1$ points, respectivement).

Les digestibilités apparentes dans le tractus digestif total ont été significativement plus élevées $(P<0,01)$ pour la ration expérimentale, respectivement (de $+7,4$ et de 8,0 points) pour la MS et la matière organique.

Le flux duodénal d'azote a été plus élevé que l'ingestion alimentaire total (tableau V), l'augmentation variant de $4 \%$ pour la ration témoin à $9 \%$ pour la ration expérimentale. L'ingestion d'azote a été supérieure avec la ration enrichie en pulpes $(+21 \mathrm{~g} / \mathrm{j})$, et le flux d'azote total à l'entrée de l'intestin a été augmenté de 34 $\mathrm{g} / \mathrm{j}$. Cette augmentation est presque entièrement expliquée par l'accroissement de l'azote bactérien dans les digesta duodénaux (+ $33 \mathrm{~g} / \mathrm{j}$ ) (tableau $\mathrm{V}$ ). De plus, la distribution de pulpes sèches a augmenté le flux duodénal de tous les acides aminés, cette augmentation étant significative excepté pour la sérine, la glycine, la cystéine et la méthionine (fig 1).

\section{DISCUSSION}

Les faibles vitesses de digestion des constituants pariétaux et leur temps de séjour relativement long dans le rumen sont des contraintes importantes à l'ingestion volontaire des fourrages de qualité médiocre chez les ruminants (Preston, 1982; Dulphy et Demarquilly, 1974, Ndlovu et BuchananSmith, 1985). II s'ensuit que les productions animales sont limitées par la faible ingestibilité des fourrages et leur qualité médiocre, et donc par l'apport insuffisant de nutriments énergétiques. Dans ce contexte, il est nécessaire de proposer une complémentation adéquate et d'en mesurer les effets.

II ressort des résultats de cet essai que la complémentation d'un ensilage d'herbe de qualité médiocre par des pulpes sèches s'est traduite par une tendance vers une augmentation de la quantité de MS ingérée de $9,3 \%$. Cependant, les pulpes ne peuvent pas être considérées comme supplément intégral à l'ensilage d'herbe, étant donné que l'apport de $2,56 \mathrm{~kg}$ de MS de 
Tableau V. Effet de la complémentation de l'ensilage d'herbe par les pulpes sèches sur l'utilisation de l'azote dans le rumen et le tractus digestif total des génisses.

\begin{tabular}{|c|c|c|c|c|}
\hline & $\begin{array}{l}\text { Ration } \\
\text { témoin }\end{array}$ & $\begin{array}{c}\text { Ration } \\
\text { expérimentale }\end{array}$ & $E T$ & $\begin{array}{c}\text { Niveau } \\
\text { signif stat } 1\end{array}$ \\
\hline$N$ total ingéré $(g / j)$ & 213 & 234 & 3,5 & $x$ \\
\hline \multicolumn{5}{|l|}{ Flux duodénaux $(\mathrm{g} / \mathrm{j})$ : } \\
\hline $\mathrm{N}$ total & 222 & 256 & 5,2 & $x x$ \\
\hline $\mathrm{N}-\mathrm{NH}_{3}$ & 11 & 9 & 0,5 & NS \\
\hline $\mathrm{N}$ non $\mathrm{NH}_{3}$ & 211 & 247 & 5,3 & $x x$ \\
\hline $\mathrm{N}$ endogène ${ }^{2}$ & 18 & 18 & - & - \\
\hline $\mathrm{N}$ bactérien & 124 & 158 & 5,4 & $x$ \\
\hline $\mathrm{N}$ alimentaire résiduel & 68 & 71 & 5,4 & NS \\
\hline Dégradabilité réelle de $\mathrm{N}$ alimentaire $(\%)$ & 68 & 69 & 2,8 & NS \\
\hline $\mathrm{g}$ de $\mathrm{N}$ bactérien $/ \mathrm{kg}$ MO fermentée & 31 & 31 & 2,5 & NS \\
\hline Digestibilité apparente ${ }^{3}(\%)$ & 52 & 50 & 1,0 & NS \\
\hline
\end{tabular}

$x: P<0,05 ; x x: P<0,01 ; x x x: P<0,001 .{ }^{1}$ Entre les 2 traitements. ${ }^{2}$ Valeurs calculées en supposant que $\mathrm{N}$ endogène équivaut à $2,7 \mathrm{~g} / \mathrm{kg} \mathrm{MS}$ duodénale. ${ }^{3}$ Tractus digestif total.

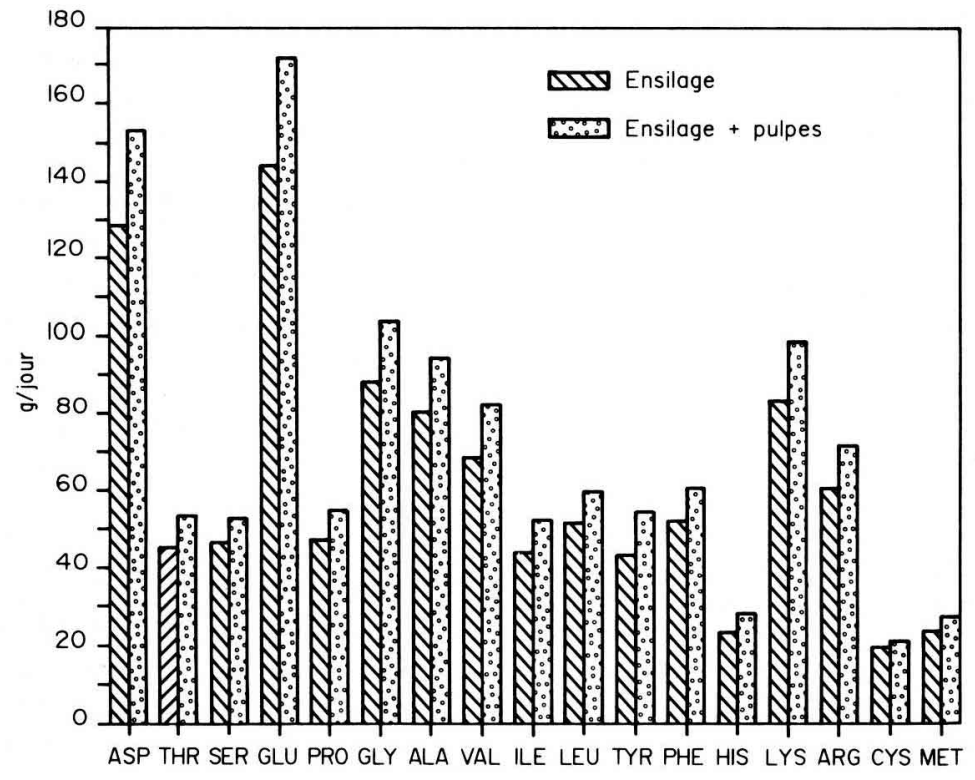

Fig 1. Effet de la complémentation d'ensilage d'herbe préfanée par des pulpes sèches sur le flux des acides aminés à l'entrée de l'intestin grêle chez des génisses (g/jour). 
pulpes a entraîné une réduction de l'ingestion d'ensilage de $1,6 \mathrm{~kg}$ de MS, soit un taux de substitution de $0,64 \mathrm{~kg}$ de $\mathrm{MS} / \mathrm{kg}$ de MS de pulpes apportées. Différents auteurs ont observé que les pulpes sèches (Castle et al, 1963; Castle, 1972) et les pulpes surpressées ensilées (Dulphy et al, 1978; De Brabander et al, 1980) réduisent l'ingestion de fourrage grossier de base et ont un effet positif sur les quantités de matière sèche totale ingérées.

Selon Muller et Beranger (1979), quand $35 \%$ de la MS totale ingérée provient des pulpes et $65 \%$ de l'ensilage d'herbe offerts aux bovins en croissance et à l'engrais, les pulpes ont une valeur d'encombrement supérieure à celle des céréales. En revanche, les essais sur vaches en milieu de lactation recevant de l'ensilage d'herbe en association avec $47 \%$ de pulpes de betteraves ou de céréales compilés par Coulon et al (1989) ont montré que ces 2 types d'aliments concentrés apportés de manière iso-énergétique modifient peu la consommation du fourrage. Lorsqu'ils sont apportés en quantités équivalentes, la consommation du fourrage avec les pulpes est en moyenne supérieure de 0,2 à $0,3 \mathrm{~kg} \mathrm{MS} / \mathrm{j}$ à celle observée avec les céréales. Les valeurs d'encombrement de ces aliments concentrés sont donc semblables aux taux usuels d'utilisation.

La supplémentation avec les pulpes sèches réduit la durée journalière de rumination malgré la teneur en constituants pariétaux, relativement élevée dans ces aliments (Welch, 1982). Ceci peut s'expliquer par la digestibilité élevée des constituants pariétaux apportés par les pulpes.

D'une façon générale, les effets des aliments concentrés sur la durée journalière de rumination sont variables. Celle-ci est réduite proportionnellement à la diminution de l'ingestion de constituants pariétaux lorsque les quantités de concentrés sont réduites. En revanche, de grandes quanti- tés de concentrés perturbent le processus de rumination. Dans le présent essai, la diminution de l'ingestion d'ensilage d'herbe préfanée après complémentation par des pulpes a entraîné une réduction de la durée unitaire de rumination et du nombre unitaire de cycles de rumination de $14 \%$ ( $P$ $<0,01)$, bien que l'ingestion de matière sèche ait été augmenté de $8,2 \%$ (tableau II). Ceci concorde avec les observations de Vérité et Journet (1973) qui ont montré que la complémentation de fourrages par des pulpes sèches réduit la durée journalière et la durée unitaire de mastication.

Les pulpes sèches de betteraves sucrières contiennent des glucides facilement fermentescibles pour les microorganismes du réticulo-rumen. La fermentation de ces glucides entraîne une diminution du $\mathrm{pH}$ moyen dans le rumen. Cette valeur reste néanmoins supérieure à 6 , qui est la valeur en dessous de laquelle l'activité cellulolytique est inhibée (Stewart, 1977). Le pH et la pression osmotique ne sont pas notablement influencés par l'addition de pulpes de betteraves séchées à l'ensilage d'herbe préfanée. En moyenne, ces valeurs correspondent à celles requises pour une activité mérycique optimale (Franzen, 1987; Welch, 1982).

Le taux de dilution de la phase liquide du rumen et la quantité de matières azotées bactériennes synthétisées par $\mathrm{kg}$ de matière organique fermentée dans le rumen (Rhode et al, 1985) n'ont pas été différents pour les 2 rations. II s'ensuit que ces 2 facteurs ne sont affectés ni par l'ingestion de matière sèche totale supérieure après complémentation de l'ensilage d'herbe préfanée par des pulpes séchées ni par l'ingestion supérieure de l'ensilage seul.

Le flux plus élevé d'azote total à l'entrée de l'intestin après complémentation de l'ensilage par des pulpes s'explique par une plus grande quantité d'azote bactérien 
(+27\%). Dans l'ensemble, la quantité d'azote total qui arrive dans le duodénum excède l'azote total ingéré avec les 2 types de ration : de $+9 \%$ et $+4 \%$ avec les pulpes et avec l'ensilage seul, respectivement. Narasimhalu et al (1989) rapportent un flux d'azote duodénal plus élevé de $2 \%$ par rapport à l'azote ingéré chez des génisses recevant un ensilage d'herbe préfanée de bonne qualité. L'efficience de la synthèse de protéines bactériennes est semblable pour les 2 rations : 30,7 et 30,9 $\mathrm{g}$ d'azote bactérien par $\mathrm{kg}$ de matière organique fermentée et 24,0 et $24,1 \mathrm{~g}$ d'azote bactérien par $\mathrm{kg}$ de matière organique réellement digérée dans le rumen, respectivement. Van Nevel et Demeyer (1977) rapportent une valeur moyenne de $32 \mathrm{~g} / \mathrm{kg}$ de matière organique fermentée. La plus grande quantité d'azote bactérien arrivant dans l'intestin grêle après complémentation de l'ensilage par des pulpes s'explique principalement par la quantité plus élevée de matière organique réellement dégradée dans le rumen $(+25 \%, P<$ 0,05 ). Enfin, les pourcentages des différents acides aminés par rapport à l'azote aminé total dans le contenu duodénal, de même que la proportion de l'azote non ammoniacal retrouvée sous forme aminée, sont semblables pour les 2 rations. Les différences observées dans les quantités journalières d'acides aminés arrivant dans l'intestin (fig 1) proviennent en grande partie de l'accroissement du flux duodénal de protéines bactériennes après complémentation de l'ensilage par des pulpes.

\section{CONCLUSION}

La substitution de pulpes sèches de betteraves sucrières à un ensilage d'herbe préfanée de coupe tardive a stimulé l'ingestion de la matière sèche totale. Le taux de substitution des pulpes à l'ensilage a été de $64 \%$ de matière sèche. Cette supplémentation avec des pulpes a été accompagnée d'une augmentation de la digestibilité apparente dans le rumen et aussi de la digestibilité apparente dans le tractus digestif total.

Si on ne tient pas compte du tourteau de soja, on peut calculer que les animaux ont consacré en moyenne 35 min à ruminer chaque $\mathrm{kg}$ de matière sèche apporté par les pulpes sèches. Cette valeur est élevée comparativement aux $59 \mathrm{~min} / \mathrm{kg}$ enregistrées pour l'ensilage d'herbe de coupe tardive; mais elle est proche de la valeur de $32 \mathrm{~min} / \mathrm{kg}$ de matière sèche obtenue pour des pulpes incorporées à des pellets d'aliments complets distribués à des génisses (Teller, données non publiées). Bien que des précisions restent à apporter à ce niveau, on peut conclure que les pulpes sèches de betteraves sucrières ont un indice de fibrosité élevé. En effet, la durée de rumination additionnée d'une durée moyenne d'ingestion de $10 \mathrm{~min} / \mathrm{kg}$ de matière sèche confèrent aux pulpes sèches un indice de mastication dépassant $30 \mathrm{~min} / \mathrm{kg}$ MS, c'est-à-dire une durée supérieure à celle recommandée par Journet (1988) chez la vache laitière.

Les pulpes ont également une valeur énergétique élevée en raison de leur digestibilité élevée. De ce fait, elles augmentent la synthèse journalière de protéines microbiennes et donc, l'apport d'acides aminés dans l'intestin grêle. L'ensemble de ces observations explique l'utilisation avantageuse, qui est observée classiquement, des pulpes sèches dans les rations d'engraissement et aussi dans celles de la vache haute productrice.

\section{REMERCIEMENTS}

Travail subventionné par l'Institut pour l'encouragement de la recherche scientifique dans l'in- 
dustrie et l'agriculture (IRSIA), rue de Crayer 6 , 1050 Bruxelles.

\section{RÉFÉRENCES}

AOAC (1960) Official Methods of Analysis (9th edn) Assoc Off Anal Chem, Washington, DC

Bartiaux-Thill N, Oger R, François E, Thewis A (1988) Courbes d'excrétion fécale de l'oxyde de chrome administré aux vaches laitières au pâturage. Définition d'un échantillonnage biquotidien des fèces. Repr Nutr Dév 28, 87-88

Bhattacharya AN, Sleiman FT (1971) Beet pulp as grain replacement for dairy cows and sheep. J Dairy Sci 54, 89-95

Buysse FX, Boucque CV (1967) Les pulpes séchées, aliment de base pour la production intensive de viande. Rev Agric Bruxelles 20, 1029-1044

Castle ME (1972) A comparative study of the feeding value of dried sugar-beet pulp for milk production. J Agric Sci Camb 78, 371377

Castle ME, Drysdale AD, Waite R, Watson JM (1963) The effect of the replacement of concentrate by roots on the intake and production of dairy cows. J Dairy Sci 30, 199207

Cochran WG, Cox GM (1957) Experimental Designs. J Wiley and Sons, New York

Cordiez E, Lambot $O$, Bienfait JM, Pondant A, Van Eenaene C (1977) La pulpe séchée de betterave sucrière. Une possibilité d'économiser les céréales dans la production de viande bovine. Rev Mond Zootech 21, 18-23

Coulon JB, Faverdin $P$, Laurent $F$, Cotto $G$ (1989) Influence de la nature de l'aliment concentré sur les performances des vaches laitières. INRA Prod Anim 2, 47-53

De Brabander DL, Aerts JV, Boucque CV, Buysse FX (1980) Influence des pulpes surpressées ensilées sur l'ingestion de fourrages grossiers, la production et la composition du lait chez la vache laitière. Rev Agric Bruxelles 33, 941-952

Dulphy JP (1971) Caractéristiques du comportement alimentaire du mouton recevant des ensilages. Ann Biol Anim Biophys Biochim 11, 287-289
Dulphy JP, Demarquilly C (1974) Étude du comportement alimentaire et mérycique du mouton recevant des fourrages verts hachés. Ann Zootech 23, 193-212

Dulphy JP, Bony J, Andrieu JP (1978) Utilisation des pulpes de betteraves surpressées par les vaches laitières. Bull Tech CRVZ Theix, INRA 34, 15-21

El-Shazly K, Hungate RE (1966) Method for measuring diaminopimelic acid in total rumen contents and its application to the estimation of bacterial growth. App/ Microbio/ 14, 27-32

Faichney GJ (1975) The use of markers to partition digestion within the gastro-intestinal tract of ruminants. In: IV Int Symp Ruminant Physiol (IW Donald, ACl Warner, eds) Univ New England Press, Armidale, NSW, Australia, 277-292

Franzen $H$ (1987) Les aspects physiologiques de l'alimentation des vaches à haute production laitière (II). Agra-magazine, novembre 1987, 19-22

Giger $S$, Thivend $P$, Sauvant $D$, Dorléans $M$, Journaix P (1987) Étude de l'influence préalable de différentes enzymes amylolytiques sur la teneur en NDF d'aliments du bétail. Ann Zootech 36, 39-48

Goering MK, Van Soest PJ (1970) Forage fiber analyses (apparatus, reagents, procedures and some applications). Agric Handbook 379, ARS, USDA, Washington, DC

Hyden SA (1955) A turbimetric method for the determination of higher polyethyleneglycols in biological material. Kungl Lantbr Högsk Ann 22, 139-145

Journet $\mathrm{M}$ (1988) Optimisation des rations. In: Alimentation des bovins, ovins et caprins (Jarrige $R$, ed) INRA, Paris, 121-133

Madsen J, Hvelplund T (1985) Protein degradation in the rumen. A comparison between in vivo, nylon bag, in vitro and buffer measurements. Acta Agric Scand (suppl) 25, 103-104

Muller A, Béranger C (1979) Utilisation des pulpes de betteraves déshydratées en complément d'ensilage d'herbe par les bovins en croissance et à l'engrais. Bull Techn CRZV, Theix, INRA 35, 53-58

Narasimhalu P, Teller E, Vanbelle M, Foulon M, Dasnoy $F$ (1989) Apparent digestibility of nitrogen in rumen and whole tract of Friesian cattle fed direct cut and wilted silages. J Dairy Sci 72, 2055-2061 
Ndlovu LR, Buchanan-Smith JG (1985) Utilization of poor quality roughages by sheep : effects of alfalfa supplementation on parameters, fiber digestion and rate of passage from the rumen. Can J Anim Sci 65, 693-703

Petry H, Rapp W (1971) Zur Problematik der Chromoxydbestimmung in Vardauungesversuchen. $Z$ Tierphysiol Tierernähr Futtermittelkde 27, 181-189

Preston TR (1982) Nutritional limitations associated with the feeding of tropical forages. $J$ Anim Sci 54, 877-884

Rhode LM, Weakley DC, Satter LD (1985) Effect of forage amount and particle size in diets of lactating dairy cows on site of digestion and microbial protein synthesis. Can $J$ Anim Sci 65, 101-111

Ruckebusch Y (1963) Recherche sur le comportement alimentaire des ruminants. Thèse de Doctorat Vétérinaire, Lyon

SAS (1982) SAS User's Guide : Statistics. SAS Inst Inc, Cary, NC

Schoch W (1949) Die bei der Trocknung von Silageproben im Trockenschrank auftretende Verluste and flüchtigen Säuren und Basen. Mitt Geb Lebensmittelunters Hyg 40, 170

Stewart CS (1977) Factors affecting the cellulolytic activity of rumen contents. Appl Environ Microbio/ 33, 497-502
Teller E, Vanbelle $M$, Kamatali $P$, Wavreille $J$ (1989) Intake of direct cut or wilted grass silage as related to chewing behavior, ruminal characteristics and site and extent of digestion by heifers. J Anim Sci 67, 2802-2809

Vanbelle M (1973) Alimentation du bétail et pulpe séchée. La sucrerie belge 92, 223-241

Van Nevel CJ, Demeyer DI (1977) Determination of rumen microbial growth in vitro from ${ }^{32}$-labelled phosphate incorporation. $\mathrm{Br} J$ Nutr 38, 101-114

Vérité R, Journet M (1973) Utilisation de quantités élevées de betteraves par les vaches laitières : Étude de l'ingestion, de la digestion et des effets sur la production. Ann Zootech 22, 219-235

Welch JG (1982) Rumination, particle size and passage from the rumen. $J$ Anim Sci 54, 885894

Welch JG, Smith AM (1971) Effect of beet pulp and citrus pulp on rumination activity. $J$ Anim Sci 33, 472-475

Whitehead R, Cooke KJ, Chapman BT (1967) Problems associated with the continuous monitoring of ammoniacal nitrogen in a river water. Automation in Analytical Chemistry. Technicon Symposia. Vol 2, New York, 337380 